\title{
Die Bioinduktionstherapie mit Imiquimod beim solitären Keratoakanthom - Eine therapeutische Alternative?
}

\author{
C. Oster-Schmidt ${ }^{1}$ \\ P. Altmeyer ${ }^{2}$ \\ M. Stücker ${ }^{2}$
}

\author{
The Bio-Induction-Therapy with Imiquimod in Solitary Keratoacanthoma - \\ A Therapeutical Alternative?
}

\section{Zusammenfassung}

Ein 78-jähriger Patient entwickelte in 6 Wochen im linken Ohr ein Keratoakanthom. Dieses wurde topisch mit täglicher Imiquimodapplikation über 4 Wochen behandelt. Innerhalb von 6 Wochen kam es zu einer kompletten Abheilung. Obwohl kontrollierte Studien zur Wirkung von Imiquimod in der Behandlung von Keratoakanthomen fehlen, legt die rasche Regression des Tumors und das gute kosmetische Resultat nahe, dass diese Therapie zukünftig eine Rolle in der Behandlung von Keratoakanthomen in Problemlokalisationen spielen könnte.

\section{Abstract}

A 78-year old male patient developed a keratoacanthoma, present on the left ear for 6 weeks. He was treated with topical application of imiquimod performed daily for four weeks. This led to complete resolution within 6 weeks. Although controlled studies on the effect of imiquimod in keratoacanthoma are not available, the rapid regression and the good cosmetic result suggest that in future this therapy may play a role in treating such lesions especially in problem locations.
Zurückgehend auf einen Vorschlag Freudenthals (zit. in [13]) wird heute noch der vermutlich erstmals vom Londoner Chirurgen G. Jonathan Hutchinson 1889 [12] beschriebene Hauttumor Keratoakanthom genannt. Die Dignität dieser Neoplasie der Haarfollikel ist nicht eindeutig geklärt. Einerseits wird sie bei den Pseudokanzerosen [3,5] eingeordnet, andererseits bei den malignen epithelialen Tumoren [1,4,18]. Andere Autoren [19] sprechen von einem benignen Prozess, der häufig maligne entartet.

Das Keratoakanthom entsteht auf klinisch unveränderter Haut innerhalb von Tagen bis wenigen Wochen. Es tritt am häufigsten solitär auf, erreicht eine durchschnittliche Größe von 10-25 mm und betrifft überwiegend lichtexponierte Areale hellhäutiger Menschen des mittleren und höheren Lebensalters. Prädilektionsstellen sind Gesicht und Nacken in ca. 75\% sowie Handrücken und Unterarme in ca. $13 \%$ der Fälle. Das männliche Geschlecht ist 2- bis 3-mal sooft befallen, Raucher häufiger als
Nichtraucher. Charakteristischerweise durchläuft das Keratoakanthom mehrere Stadien. Die Involution mit Ausbildung einer Narbe, die wegen Substanzverlust schüsselförmig eingesenkt und häufig hyperpigmentiert sein kann, woraus eine kosmetische Auffälligkeit resultiert, kann 4 Monate oder wesentlicher länger dauern. Differenzialdiagnostisch gilt es abzugrenzen: spinozelluläres Karzinom, Basaliom, Molluscum contagiosum, Verruca vulgaris, hypertrophe aktinische Keratose, Trichoepitheliom und atypisches Fibroxanthom [1,3,13]. Die Therapie der Wahl ist die Exzision mit mikrografischer Schnittrandkontrolle. Daneben werden verschiedene so genannte blinde Therapiemodalitäten empfohlen. Hierzu zählt z.B. die intraläsionale Applikation von Interferonen. Im folgenden Fall berichten wir über die erfolgreiche topische Behandlung eines solitären Keratoakanthoms mit einer $5 \%$ igen Imiquimodcreme. 


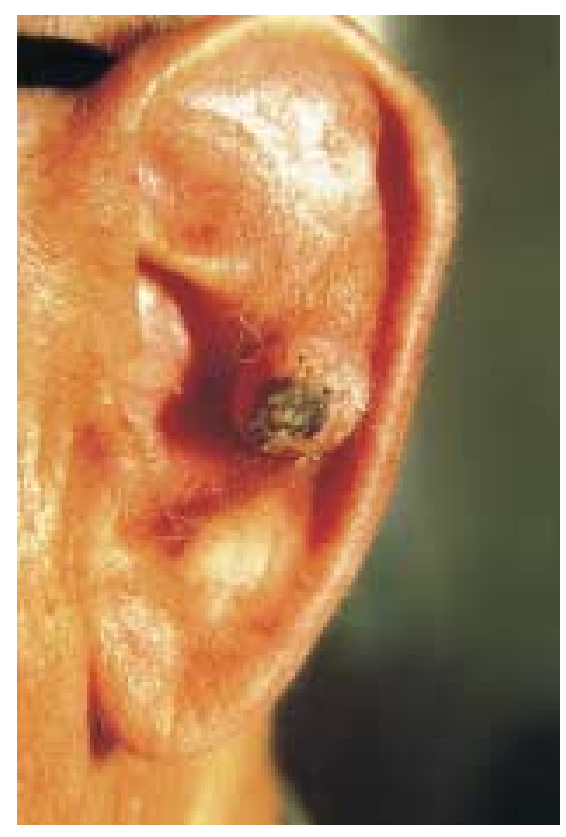

Abb. 1 Keratoakanthom. Tumor vor Beginn der topischen Imiquimodapplikation.

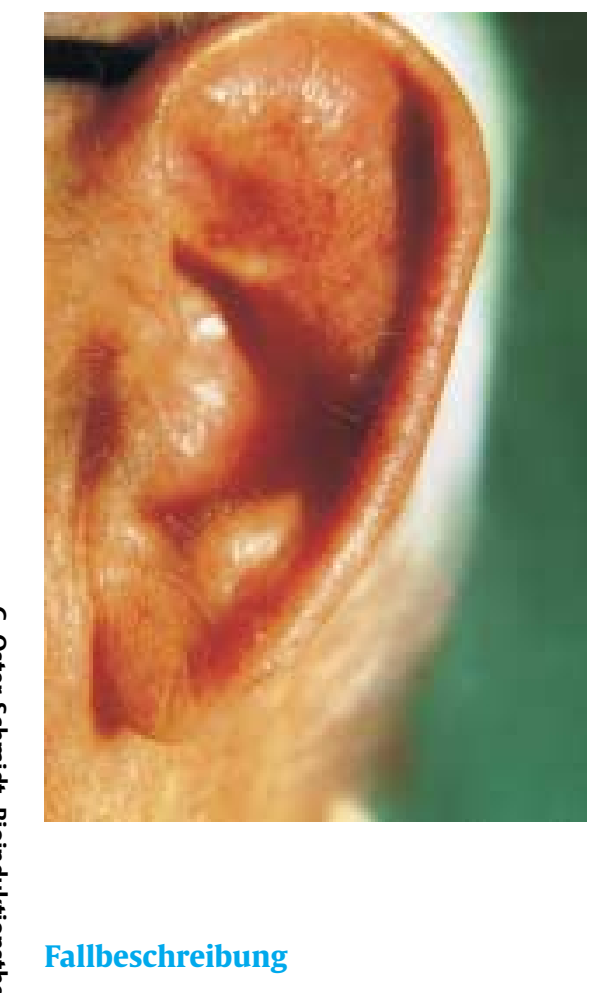

Abb. 3 Keratoakanthom. Komplette narbenfreie Abheilung 6 Wochen nach Therapiebeginn.

\section{Anamnese}

Bei dem 78-jährigen Nichtraucher war es innerhalb von 6 Wochen zur Bildung eines $12 \mathrm{~mm}$ großen, hautfarbenen, scharf abgegrenzten, kalottenförmigen Tumors mit zentralem keratotischen Pfropf in der Scapha der linken Ohrmuschel gegenüber dem Porus acusticus externus (Abb.1) gekommen, Beschwerden werden nicht geäußert.

\section{Therapie und Verlauf}

Aufgrund der klinisch gestellten Diagnose erfolgte die topische Therapie mit dem Immunmodulator Imiquimod im Sinne eines Heilversuches, nachdem ein operatives Vorgehen seitens des Patienten strikt abgelehnt worden war. Das Therapieregime sah die tägliche Selbstapplikation der 5\%igen Imiquimodcreme über 10 Stunden unter Okklusionsbedingungen (Abkleben mit einem

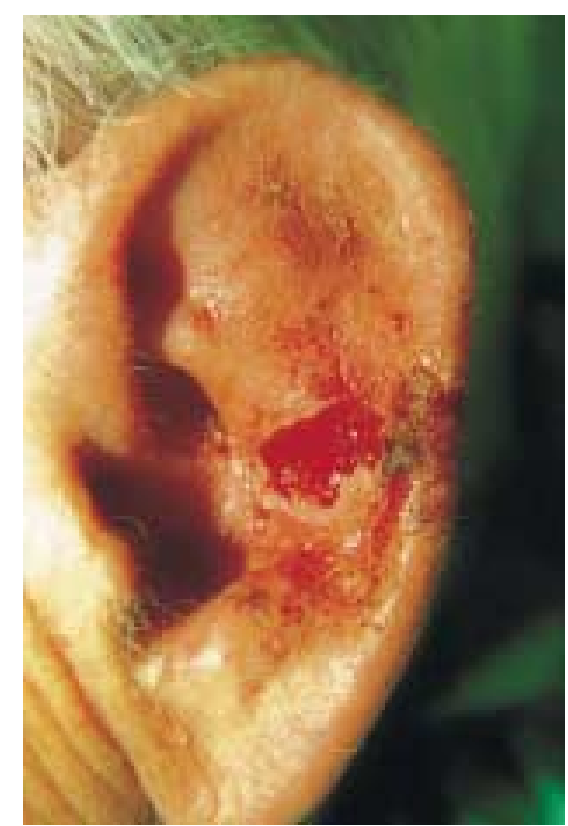

Abb. 2 Keratoakanthom. Tumor nach 4-wöchiger täglicher Therapie und Entfernung aus dem Tumorbett.

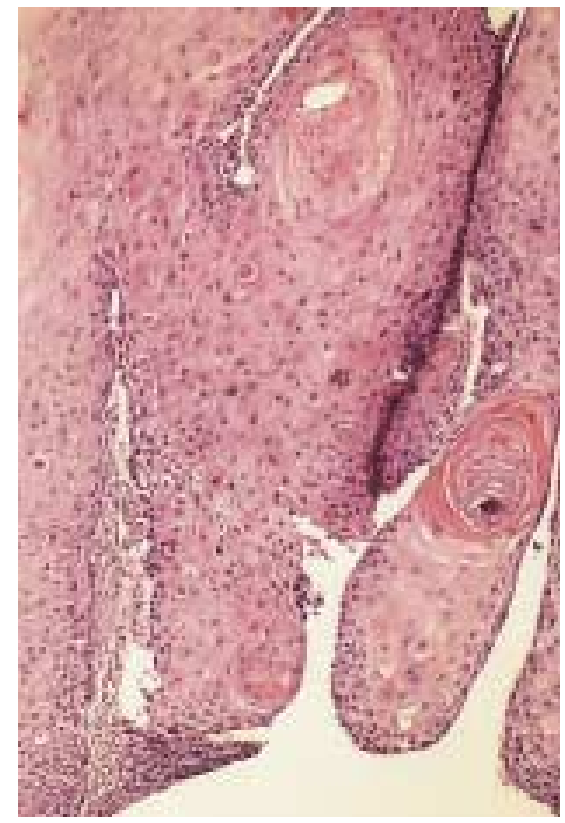

Abb. 4 Keratoakanthom. Histologisches Bild des Tumors. HE.

wasserfesten Pflaster [Hansaplast Aquaprotect, Beiersdorf, Hamburg, Deutschland]) vor. Bereits nach 2 Wochen waren Anteile des Tumorrandbereichs zerfallen und ließen sich problemlos mit einer Pinzette entfernen. Weitere 2 Wochen danach konnte der Tumor ohne Anästhesie schmerzlos mit einer Pinzette aus dem Tumorbett herausgezogen werden. Dabei kam es zu einer kurzzeitigen Blutung (Abb. 2). Der Tumor wurde der histologischen Aufarbeitung zugeführt. Nach insgesamt 6 Wochen war die entstandene Läsion komplett narbenfrei abgeheilt (Abb. 3). Seit einem Jahr ist der Patient rezidivfrei.

\section{Histologie}

Epithelialer Tumor mit kräftigen Hyperkeratosen, zum Teil pfropfartig angeordnet und von Proliferaten epithelialer Zellen umrandet. Zur Oberfläche hin Papillomatose, zur Tiefe hin Proliferate polymorpher epithelialer Zellen mit mäßiger Zell- und Kernpolymorphie mit unterschiedlichen Kernformen, -größen 
und -färbeverhalten. Vermehrte Mitosen (Abb. 4). PCR auf Material von humanen Papillomaviren negativ. Diagnose: Keratoakanthom.

\section{Besprechung}

Aufgrund der Tendenz von Keratoakanthomen zur Spontanregresssion könnte eine zuwartende Haltung in Bezug auf das therapeutische Prozedere eingenommen werden. Dies erscheint jedoch aus diversen Gründen nicht sinnvoll: Aus Sicht des Patienten ist es unzumutbar, bei den vor allem im Gesicht lokalisierten Keratoakanthomen deren Monate dauernden Selbstheilungsprozess abzuwarten, der zudem mit einer kosmetisch unbefriedigenden Narbenbildung einhergehen kann; aus der Sicht des Arztes ist es problematisch, ein spinozelluläres Karzinom klinisch abzugrenzen, so dass eine histologische Aufarbeitung zwingend ist. Auf jeden Fall ist bei Keratoakanthomen auf der Nase, am Augenlid oder in der Ohrmuschel ein aktives Vorgehen wegen der drohenden Perforationsgefahr indiziert. Dies gilt auch für solche bei betagten Menschen, weil bekannt ist, dass bei ihnen die Keratoakanthome ein ausgesprochen aggressives Wachstumsverhalten an den Tag legen respektive nicht selten maligne degeneriert sind [19] und somit, wenn auch selten, metastasieren können. Solange die Dignität des Keratoakanthoms nicht zweifelsfrei geklärt ist, ist eine histologische Verifizierung der klinischen Diagnose zu fordern. Als wichtigster Auslöser für die Entstehung von Keratoakanthomen gilt neben chemischen Karzinogenen (Teer, Pech) und Immunsuppression (AIDS-Kranke, Transplantierte) das UV-Spektrum des Lichtes. Welche Rolle humanen Papillomaviren zukommt, ist noch unklar, obgleich mehrfach HPVDNA nachgewiesen werden konnte $[9,15]$. Für die Behandlung von Keratoakanthomen existieren verschiedene Verfahren, die lokalisationsabhängig zum Einsatz kommen. Therapie der ersten Wahl ist die chirurgische Exzision, die teilweise als plastischer Eingriff aufgrund des entstandenen Gewebedefektes vorgenommen werden muss. Neben der Infektionsgefahr besteht in diesen Fällen das Risiko der postoperativ auftretenden kosmetisch störenden und psychisch belastenden Narbenbildung. Um dies zu reduzieren, werden folgende Methoden, die unter dem Begriff der blinden Therapiemodalitäten subsumiert werden, nach vorausgehender Diagnosesicherung der Schnittbiopsie eingesetzt: Kürettage, Kauterisation, Kryotherapie, Radiatio [7], intraläsionale Applikation von Interferon alpha und gamma [11], von Methotrexat $[6,17]$, von 5-Fluorouracil $[8,10]$ sowie von Bleomycin [2]. Wir verzichteten in unserem Fall auf die intraläsionale Injektion eines der aufgeführten Zytostatika. Die Idee, Imiquimod als die Immunantwort modifizierende Substanz einzusetzen, basiert auf dem Faktum, dass diese topisch zu applizierende Substanz die zellmediierte Immunantwort mittels Induktion von (hauptsächlich) TH1-Zytokinen, neben TNF alpha und verschiedenen Interleukinen auch Interferon alpha [20,22], aktiviert. Letzteres stimuliert direkt natürliche Killerzellen, welche ihrerseits virusinfizierte und Tumorzellen abtöten. Diesem Verfahren geben wir die Bezeichnung „Bioinduktionstherapie“. Der Behandlungsverlauf bei unserem Patienten bestätigte eindrucksvoll den theoretischen Ansatz. Hierzu passen auch erfolgreiche Behandlungen von Basalzellkarzinomen, initialen Plattenepithelkarzinomen und aktinischen Keratosen [14,16,21]. Somit stellt die Imiquimodapplikation eine weitere Möglichkeit in der Therapie von Keratoakanthomen für problematische Lokalisationen dar. Sie lässt im Gegensatz zu allen anderen blinden Therapiemodalitäten (Ausnahme Kürettage) eine histologische Auf- arbeitung nach Therapieende zu. In der Zwischenzeit sind zwei weitere Patienten mit einem Keratoakanthom (frontal bzw. auf dem Capillitium lokalisiert) erfolgreich mit der Bioinduktionstherapie behandelt worden. Das Nebenwirkungsspektrum war für alle Patienten tolerabel: Pruritus und Brennen wurden angegeben. Der Nachbeobachtungszeitraum umfasst 12 bzw. 6 Monate, die kritische Phase, in der die meisten Rezidive auftreten. Zusammenfassend darf gesagt werden, dass die Bioinduktionstherapie mit 5\%iger Imiquimodcreme durchaus eine Bereicherung des Therapiespektrums für Keratoakanthome darstellt, weil sie komfortabel für den Patienten ist, ein hohes Maß an Sicherheit gewährleistet und zu guten kosmetischen Resultaten führt. Die an drei Patienten gewonnenen ersten positiven Erfahrungen bedürfen der Bestätigung durch kontrollierte Studien.

\section{Danksagung}

Unser Dank gilt Frau Dr. med. P. Kammann der Firma 3M Medica (Borken), die uns freundlicherweise das Präparat Imiquimod (Aldara) zur Verfügung gestellt hat.

\section{Literatur}

${ }^{1}$ Ackerman AB, Kerl H, Sanchez J. A clinical atlas of 101 common skin diseases with histopathologic correlation. New York: Ardor Scribendi, 2000

${ }^{2}$ Andreassi A, et al. Guess what! Keratoacanthoma treated with intralesional bleomycin. Eur J Dermatol 1999; 9: 403-405

3 Braun-Falco O, Plewig G, Wolff HH, Burgdorf WHC. Dermatology. 2nd completely revised ed. Berlin, Heidelberg, New York: Springer, 2000

${ }^{4}$ Braun-Falco O, Plewig G, Wolff HH. Dermatologie und Venerologie. 4. Aufl. Berlin, Heidelberg, New York: Springer, 1997

${ }^{5}$ Champion RH, Burton JL, Burns DA, Breathnach SM. Rook/Wilkinson/ Ebling. Textbook of dermatology in 4 volumes. 6th ed. Oxford, London: Blackwell Science, 1998

${ }^{6}$ Cuesta-Romero C, et al. Intralesional methotrexate in solitary keratoacanthoma. Arch Dermatol 1998; 134: 513-514

${ }^{7}$ Donahue B, et al. Treatment of aggressive keratoacanthomas by radiotherapy. J Am Acad Dermatol 1990; 23: 489-493

${ }^{8}$ Ebner H, et al. Lokalbehandlung des Keratoakanthoms mit 5-Fluorouracil. Hautarzt 1975; 26: 585-588

${ }^{9}$ Gassenmaier A, et al. Human papillomavirus 25-related DNA in solitary keratoacanthoma. Arch Dermatol Res 1986; 279: 73 - 76

${ }^{10}$ Goette DK, et al. Successful treatment of keratoacanthoma with intralesional fluorouracil. J Am Acad Dermatol 1980; 2: 212 -216

${ }^{11} \mathrm{Grob} J$ J, et al. Large keratoacanthomas treated with intralesional interferon alfa-2a. J Am Acad Dermatol 1993; 29: 237-241

12 Hutchinson J. Morbid growths and tumours I. The „crateriform ulcer of the face“, a form of acute epithelial cancer. Trans Path Soc Lond 1889; 40: $275-281$

${ }^{13}$ Korting GW. Die Haut im Alter und ihre Krankheiten. Stuttgart, New York: Schattauer, 1973

${ }^{14}$ Mackenzie-Wood A, Kossard S, de Launey J, Wilkinson B, Owens ML. Imiquimod 5\% cream in the treatment of Bowen's disease. J Am Acad Dermatol 2001; 44: 462-470

${ }^{15}$ Magee KL, et al. Human papillomavirus associated with keratoacanthoma. Arch Dermatol 1989; 125: 1587-1589

${ }^{16}$ Marks R, Gebauer K, Shumack S, Amies M, Bryden J, Fox TL, Owens ML. Imiquimod $5 \%$ cream in the treatment of superficial basal cell carcinoma: Results of a multicenter 6-week dose-response trial. J Am Acad Dermatol 2001; 44: 807-813

${ }^{17}$ Remling R, et al. Intraläsionale Methotrexat-Injektion - Eine wirkungsvolle, zeit- und kostensparende Therapiealternative bei operativ schwierig zu behandelnden Keratoakanthomen. Hautarzt 2000; 51: $612-614$

${ }^{18}$ Sams WM, Lynch PJ. Principles and Practice of Dermatology. 2nd ed. New York: Churchill Livingstone, 1996 
${ }^{19}$ Sanchez-Yus E, et al. Solitary keratoacanthoma - A self-healing proliferation that frequently becomes malignant. Am J Dermatopathol 2000; 22: $205-210$

${ }^{20}$ Testerman TL, et al. Cytokine induction by the immunomodulators imiquimod and S-27609. J Leukoc Biol 1995; 58: 365-372
${ }^{21}$ Stockfleth E, Meyer T, Benninghoff B, Christophers E. Successful treatment of actinic keratosis with imiquimod cream $5 \%$ a report of six cases. Br J Dermatol 2001; 144: 1050-1053

22 Wagner TL, et al. Modulation of TH1 and TH2 cytokine production with the immune response modifiers R-848 and imiquimod. Cell Immunol 1999; 191: 10-19

\section{Buchbesprechung}

Fortschritte der praktischen Dermatologie und Venerologie. Vorträge und Dia-Klinik der 17. Fortbildungswoche 2000.

Fortbildungswoche für Praktische Dermatologie und Venerologie e.V. c/o Klinik und Poliklinik für Dermatologie.

Plewig, G., K. Degitz (Hrsg.)

685 Seiten, 381 Abb., 137 Tab. Springer, Berlin, Heidelberg 2001. Geb. DM 199,90. ISBN 3540-66650-8

Es handelt sich um den Berichtsband der 17. Fortbildungswoche in München vom Juli 2000 an welcher 1650 Dermatologen von nah und fern aktiv und passiv teilgenommen haben. Diese Woche war ein Erfolg und krönte die Serie, die 1951 in wesentlich bescheidenerem Rahmen begann. Der Berichtsband umfasst 685 Seiten und ist vom Springer-Verlag als vollwertiges, dauerhaftes Buch dargeboten. Die verdienstvollen Herausgeber, Gerd Plewig und sein Mitarbeiter Klaus Degitz, haben denn auch ein hervorragendes Werk vorzulegen, das die Fortbildungswoche meisterlich spiegelt. Was dem Besucher wegen der parallelen Veranstaltungen nicht möglich war, vermag das Buch nachzuliefern; die vollständige und wiederholbare Information eines jeden Themas, wann es beliebt. Die Aufmachung wird vom Gehalt bestens ergänzt und dieser steht in nichts zurück. Es wird eine das Fach abdeckende, auch die speziellen und eher randständigen Gebiete erfassende Übersicht über unser Fach, das Wissen, die Diagnostik, die Therapie (evident und empirisch) und auch die Forschungsbemühungen dargeboten und es kommen noch Einblicke in Nachbarfächer dazu, gleichsam Blicke über den Zaun. Es ist auch die Diaklinik in Text und Farbbild enthalten. Die Berichte über die Kurse und Seminare sind vorhanden, erfreulich offen und aussagestark. Natürlich gibt es Themen, zu welchen dieses Jahr mehr Neuigkeiten zu berichten und vorzustellen sind, als zu anderen. Dies wechselt fortschrittskonform immer wieder mit den Jahren. Aber es sind alle Gebiete, auch solche die zu stagnieren scheinen, gut zusammengefasst und gemäß dem „Stand der Zeit“ versehen. So wird das Buch auch im Alltag gebraucht werden können, oft und immer wieder zu besonderen Fragen oder zur Übersicht. Es kann die Praxisnähe bieten. Es wird Bestand haben und halten, mindestens so lange bis Gerd Plewig den nächsten Fortbildungskurs durchführt und in einem ebenso prächtigen wie umfangreichen Band dokumentiert. Bis dahin ist dem vorliegenden Band der stete Gebrauch und damit der Nutzen für uns Dermatologen sicher.

E. G. Jung, Heidelberg 